\title{
Genome-wide association study for SNPs associated with PCOS in human patients
}

\author{
LI CHEN $^{1}$, LING-MIN HU ${ }^{1}$, YU-FENG WANG ${ }^{1}$, HAI-YAN YANG $^{1}$, XIAO-YANG HUANG ${ }^{1}$, \\ WEI ZHOU ${ }^{1}$ and HAI-XIANG SUN ${ }^{2}$
}

${ }^{1}$ The Reproductive Medicine Center of Changzhou Maternal and Child Health Care Hospital, Changzhou, Jiangsu 213003;
${ }^{2}$ The Reproductive Medicine Center of Nanjing Gulou Hospital, Nanjing, Jiangsu 210008, P.R. China

Received January 30, 2017; Accepted May 25, 2017

DOI: $10.3892 / \mathrm{etm} .2017 .5113$

\begin{abstract}
This study investigated the possible association between single nucleotide polymorphism (SNP) sites on a genome wide level and the presence of polycystic ovary syndrome (PCOS) in a local population. Patients treated for PCOS in the outpatient clinic of the reproductive medicine center of Changzhou Maternal and Child Health Care Hospital (affiliated to Nanjing Medical University) from January of 2010 to December 2012 were selected. Female patients affected by infertility due to simple oviduct reasons or male factors, during the same period, were enrolled for the control group. A genome-wide association study was performed. Specific experimental steps included extraction of the total human DNA and optimization of PCR amplification of target genes; flight mass spectrometry for genotyping; and statistical analyses of sequencing results. By primary selection and secondary verification at two stages in the experiment, three SNP sites were found to contain significantly different allele frequencies between the patient and control groups $(\mathrm{P}<0.05)$ : rs346795081 on THADA, rs346803513 on DENND1A and rs346999236 on TOX3. The average expression levels at the three discovered SNPs sites were significantly different between the patient and the control groups, indicating their correlation with PCOS, and the possible role of their corresponding genes on the pathogenesis of the disease.
\end{abstract}

\section{Introduction}

Polycystic ovary syndrome (PCOS) is a female endocrine disease in which high levels of androgen are produced. Symptoms include abnormalities of menstruation, hirsutism, acne, pelvic pain and difficulty achieving pregnancy (1-3).

Correspondence to: Dr Hai-Xiang Sun, The Reproductive Medicine Center of Nanjing Gulou Hospital, 321 Zhongshan Road, Nanjing, Jiangsu 210008, P.R. China

E-mail: bavk2878@163.com

Key words: genome-wide association study, polycystic ovary syndrome, genetics
Patients may even present diabetes mellitus, obesity, obstructive sleep apnea, heart disease, emotional disorders, endometrial carcinoma and other complications $(4,5)$. The pathogenesis mechanisms of PCOS are not yet clear. In 2013, a summary on the pathogenesis of PCOS found the incidence of PCOS not only being influenced by the living environment of patients, but also by genetics (6). Later, a study group found a possible influence of IL-6 in pig ovary cells in the development of PCOS. Their experiment results suggest that IL-6 may influence the expression of key mRNAs in ovarian cells (7). Studies on the genetic influence on PCOS are still lacking; this research aimed at discovering associations to SNPs in a wide genome analysis.

Genome-wide association studies (GWAS) are commonly applied to analyze the correlation between DNA polymorphisms and specific disease traits. Such analyses allow one to investigate the distribution characteristics of alleles in large population groups. GWAS usually focuses on the correlation characteristics for single nucleotide polymorphisms (SNPs). Patients in the experiment group were classified according to the clinical manifestations, while the genotype was not given priority. Each participant provided a DNA sample and millions of genetic variations were read by SNP arrays. SNP variations found more frequently in affected individuals than in controls were chosen for further analyses (8-11). In recent years, researchers have investigated the correlation between genomes and diseases $(12,13)$. The use of GWAS integrates molecular biology, genetics and statistics and may reveal pathogenic mechanisms of diseases at a molecular level. This investigation adopted the GWAS method to gather information on SNPs that may be associated with PCOS and may help elucidate pathogenic mechanisms of the disease.

\section{Materials and methods}

To confirm the PCOS diagnosis, previous experiments also detected the prolactin, luteinizing hormone and insulin resistance levels in patients. According to the Rotterdam standard proposed by the European Society for Human Reproduction and Embryology (14), persons with over two items exceeding the normal level were defined as having PCOS. During the first experimental stage 200 female PCOS patients who came to the hospital for treatment were enrolled in the study. The 
age of the patients ranged from 22 to 30 years, averaging $28.3 \pm 3.6$ years. The age of the 228 healthy individuals enrolled in the control group ranged from 19 to 33 years, averaging $25.7 \pm 5.3$ years. At the 2 nd experimental stage, 200 further female PCOS patients who came to other hospitals in the same province were selected. The patient ages ranged from 19 to 33 years, averaging $25.7 \pm 5.3$ years. The ages of 1,400 healthy individuals in the control group ranged from 19 to 35 years, averaging $26.6 \pm 4.9$ years.

Inclusion criteria for the PCOS group: the diagnosis of PCOS conformed to the Rotterdam standards proposed in 2003, details are as follows: i) infrequent menstruation or amenorrhea; ii) clinical or biochemical manifestations of hyperandrogenism; and iii) vaginal ultrasonography revealed at least one ovary with $>12$ follicles of $2-8 \mathrm{~mm}$. Patients who met two of the above conditions and had other diseases, like adrenocortical hyperplasia, Cushing's syndrome, and androgen secreting tumors excluded were considered to be affected with PCOS.

Inclusion criteria for the control group: i) normal menstrual cycle; ii) normal basic endocrine detection results; iii) vaginal ultrasonography revealed no organic lesions at uterus or ovary (and the ovary showed no polycystic manifestations); iv) no administration of hormonal medicines within three months and other endocrine abnormalities could be excluded; and v) no family history of diabetes.

Blood collection. On the 3 rd to 5 th day of the menstrual cycle, elbow vein blood was extracted for determinations of hormone levels, biochemical indexes and an oral glucose tolerance test (OGTT). Height, weight, waistline and hipline were measured on the day of blood extraction. The waistline was the maximum abdomen perimeter at the level of the navel. Hipline was the maximum hip perimeter, within the space from the waist to the thigh. The body mass index (BMI) was calculated as the weight $(\mathrm{kg}) /$ height $^{2}\left(\mathrm{~m}^{2}\right)$. The weight hip ratio (WHR) was calculated as the measurements of the waistline/hipline. The homeostatic model assessment and insulin resistance (HOMA-IR) was calculated as [fasting insulin $(\mathrm{mU} / \mathrm{l}) \mathrm{x}$ fasting blood glucose $(\mathrm{mmol} / \mathrm{l})] / 22.5$. Finally, every research subject signed an informed consent form.

Blood samples of $5 \mathrm{ml}$ venous blood in EDTA were collected from each participant. The samples were centrifuged for $4 \mathrm{~h}$ according to standard methods to, respectively, separate plasma, white and red cells into $1.5 \mathrm{ml}$ centrifuge tubes. Samples not immediately processed were stored at $-80^{\circ} \mathrm{C}$ with cryopreservation for future use.

Genome extraction. DNA extraction kits (Qiagen, Hilden, Germany) were used to extract genomic DNA. This method produces a high yield, is easy and time-saving and needs only low consumption of cells (only $200 \mu \mathrm{l}$ of the WBC layer were used). The DNA concentration yields ranged between 20 and $50 \mathrm{ng} / \mu \mathrm{l}$ and their purity (measured with ultraviolet 260 OD/280 OD) was between 1.6 and 2.0, meeting research needs.

$P C R$ reaction. DNA was extracted from blood samples of research subjects, and amplicons were generated by PCR using published primers and protocols (15). The sequence of the primers used is shown in Table I.
Table I. PCR primers.

\begin{tabular}{ll}
\hline Primer name & \multicolumn{1}{c}{ Sequence } \\
\hline $1-\mathrm{F}$ & AGTCGATGATGCTAGCTGA \\
$1-\mathrm{R}$ & CGTAGCTAGCTAGCTACG \\
$2-\mathrm{F}$ & CTAGCTAGATAGCTAGCTACG \\
$2-\mathrm{R}$ & CGATGCATATTAGCTACGATGC
\end{tabular}

F, forward; R, reverse.

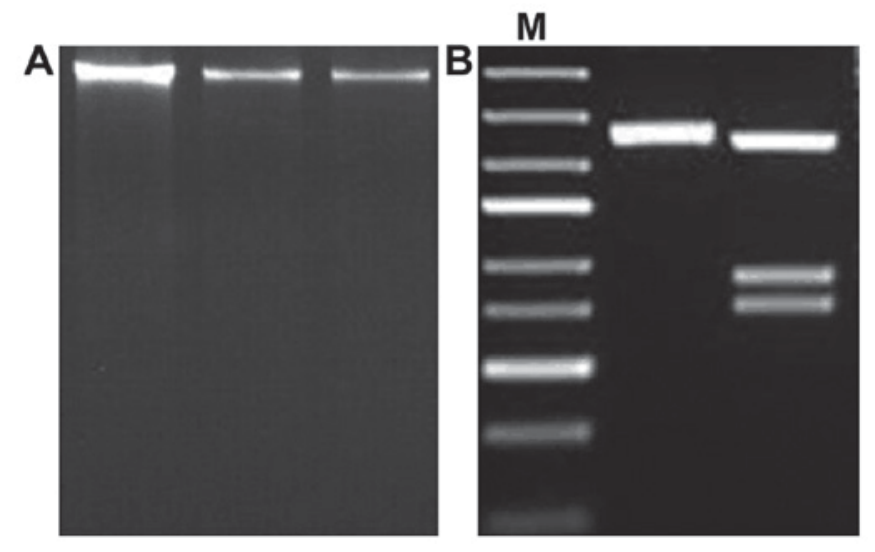

Figure 1. DNA electrophoresis of whole DNA and PCR amplicons. (A) Total genome samples of control group subjects showing successful DNA extraction. (B) PCR amplification, patient group samples, after optimization of conditions, the electrophoretic bands are clear and conform to the expected sizes. M, size marker.

SNP selection. The selection of SNP sites was completed with a Human Mapping 6.0 chip according to published methods (16). The chip included over 900,000 SNP sites of genetic variation. The primary screening was performed with a matrix-assisted laser desorption/ionization time of flight mass spectrometry (MALDI-TOF-MS). The genotype of SNPs was read according to the published report (16), after hybridization with the gene chip.

Statistical analysis. The SAS software version 9.0. (SAS Institute Inc., Cary, NC, USA) was used for statistical analyses, according to the analysis methods proposed in the literature (17).

\section{Results}

Extraction and PCR amplification of genomes. A GWAS was performed. A stable PCR amplification method as established by adjusting the denaturation temperature to $94^{\circ} \mathrm{C}$, the annealing temperature to $45^{\circ} \mathrm{C}$ and the cycle number to 35 times. The optimal PCR results can be seen in Fig. 1, as well as gel electrophoresis of whole DNA samples extracted.

Analyses of SNP sites related to PCOS during the 1st experimental stage. Ionization time-of-flight mass spectrometry was used to analyze the products of PCR amplification and its allele genes are shown in the distribution plot of Fig. 2 . 
Table II. Correlation between 10 SNP sites and PCOS by comparison of allele frequency between patients and control groups.

\begin{tabular}{llcccr}
\hline & & & \multicolumn{2}{c}{ Allele frequency } & \\
SNPs & Gene & Allele & PCOS group & Control group & P-value \\
\hline rs346795081 & THADA & $\mathrm{A} / \mathrm{C}$ & 0.426 & 0.358 & $<0.05$ \\
rs346796267 & THADA & $\mathrm{C} / \mathrm{G}$ & 0.343 & 0.339 & $>0.05$ \\
rs346797283 & DENND1A & $\mathrm{A} / \mathrm{C}$ & 0.324 & 0.321 & $>0.05$ \\
rs346803513 & DENND1A & $\mathrm{A} / \mathrm{T}$ & 0.447 & 0.412 & $<0.05$ \\
rs346982406 & DENND1A & $\mathrm{A} / \mathrm{T}$ & 0.128 & 0.125 & $>0.05$ \\
rs346996092 & TOX3 & $\mathrm{C} / \mathrm{T}$ & 0.268 & 0.257 & $>0.05$ \\
rs346999236 & TOX3 & $\mathrm{C} / \mathrm{T}$ & 0.321 & 0.295 & $<0.05$ \\
rs347021263 & YAP1 & $\mathrm{A} / \mathrm{T}$ & 0.179 & 0.186 & $>0.05$ \\
rs347026725 & YAP1 & $\mathrm{A} / \mathrm{T}$ & 0.351 & 0.350 & $>0.05$ \\
rs347029500 & YAP1 & $\mathrm{C} / \mathrm{T}$ & 0.342 & 0.325 & $<0.05$ \\
\hline
\end{tabular}

$\mathrm{P}<0.05$, statistically significant differences. SNPs, single nucleotide polymorphisms; PCOS, presence of polycystic ovary syndrome.

Table III. Correlation between the four SNP sites and the occurrence of PCOS.

\begin{tabular}{llccr}
\hline & & \multicolumn{2}{c}{ Allele frequency } & \\
\cline { 3 - 4 } SNPs & Research stage & PCOS group & Control group & P-value \\
\hline rs346795081 & 1st stage & 0.426 & 0.358 & $<0.05$ \\
& 2nd stage & 0.433 & 0.321 & $<0.05$ \\
Total & 0.429 & 0.339 & $<0.05$ \\
rs346803513 & 1st stage & 0.447 & 0.412 & $<0.05$ \\
& 2nd stage & 0.451 & 0.425 & $<0.05$ \\
Total & 0.449 & 0.418 & $<0.05$ \\
rs346999236 & 1st stage & 0.321 & 0.295 & $<0.05$ \\
& 2nd stage & 0.317 & 0.286 & $<0.05$ \\
Total & 0.319 & 0.290 & $<0.05$ \\
& 1st stage & 0.342 & 0.325 & $<0.05$ \\
2nd stage & 0.333 & 0.328 & $>0.05$ \\
& Total & 0.337 & 0.326 & $>0.05$ \\
\hline
\end{tabular}

SNPs, single nucleotide polymorphisms; PCOS, presence of polycystic ovary syndrome.

Firstly, after reviewing relevant literature and NCBI data, 10 SNP sites that may correlate with PCOS were selected and then verified by mass spectrometry as shown in Table II.

The table shows that among 10 SNP sites, only frequencies of alleles at rs346795081 on THADA gene, rs346803513 on DENND1A gene, rs346999236 on the TOX3 gene and rs347029500 on the YAP1 gene were significantly different between the patient and control groups $(\mathrm{P}<0.05)$. Fig. 3 shows a graph of the relative frequencies for all the 10 alleles in the patient and control groups.

Sample amplification verification during the 2 nd stage. Four SNP sites were selected during the 1st stage. These sites were deemed relevant to the pathogenesis of PCOS. To verify the accuracy of the allele selection, PCR was used to amplify the same regions from control and DNA samples of PCOS patients. A correlation analysis was carried out to find a link between the occurrence of the four SNP sites and PCOS, and the results are shown in Table III.

The sample size was increased to 1,200 cases during the 2 nd stage of the study. Results show the correlation between rs347029500 and PCOS had a P>0.05, which rules out statistical significance. Considering the results of the 1st and 2nd experimental stages, only the differences of allele frequencies at three SNP sites, that is, rs346795081, rs346803513 and rs346999236 were still significantly different $(\mathrm{P}<0.05)$. Fig. 4 shows the differences between the allele frequencies in the patient and control groups are only significant at rs346795081, rs346803513 and rs346999236 $(\mathrm{P}<0.05)$. 


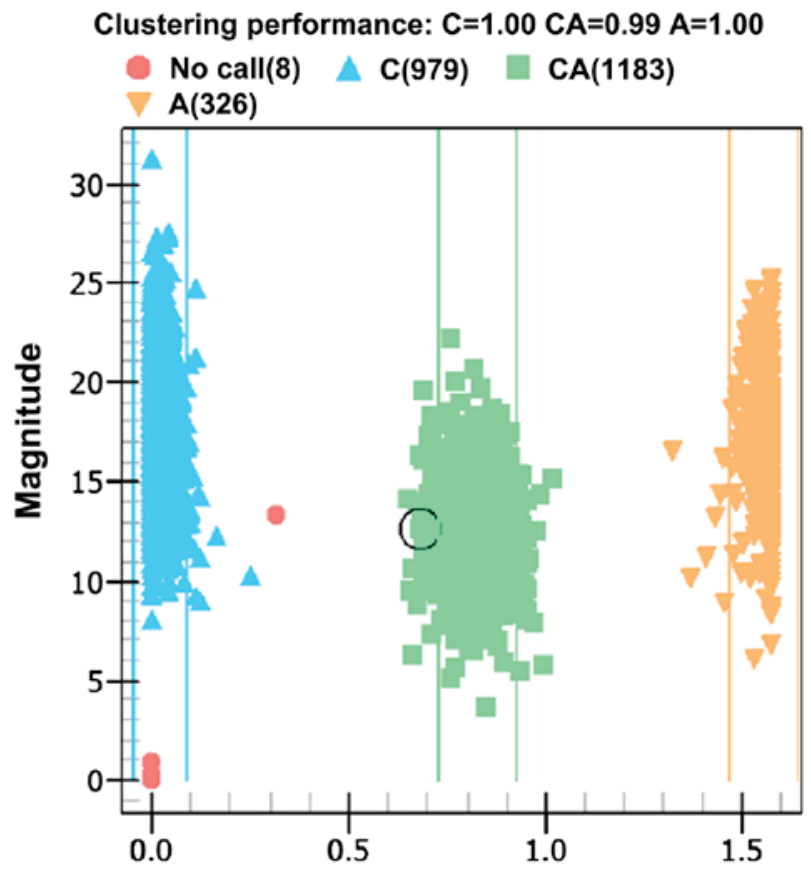

Figure 2. Plot of allelic distribution by time-of-flight mass spectrometry. The triangles indicate homozygous genes. The squares indicate heterozygous genes. The circles indicate unknown types.

\section{Discussion}

With the current scientific and technological developments, the third generation sequencing technology has made gene sequencing no longer a difficult problem. High throughput and highly effective sequencing can often provide abundant information (18). Ensuring that the processing and analysis of the data obtained give accurate results has become a biggest challenge. GWAS are based on high throughput sequencing and are combined with statistics to select and sort the large amount of data got from sequencing (19). GWAS can thus predict or help infer effective information and instruct on the research direction (20). This study detected SNPs that may be correlated with the manifestation of PCOS GWAS, the selected SNPs will help lay a foundation for future experiments elucidating the role of different genes in the pathogenesis of PCOS (21).

The experiment was divided into four parts. The first part involved the selection of patients and the acquisition of DNA from blood samples by solid phase extraction. Protein components with strong polarity were removed first and then the purity of the DNA after elution of solid phase extraction column was determined by electrophoresis. Next, the optimal parameters for PCR were established.

For genotyping, the third part of the study, ionization time-of-flight mass spectrometry was adopted. Different SNP sites are found in fragments of different length. The nucleotide length defines the molecular weight. Spectrometry identifies SNP sites by difference in molecular weight.

There is literature indicating that the frequency of SNP alleles in the patient group vs. the frequency in the control group can imply a correlation with the patient's disease (17). We compared the gene frequency at different SNP sites between the PCOS and the control group and selected SNP sites that are significantly different between the two groups.

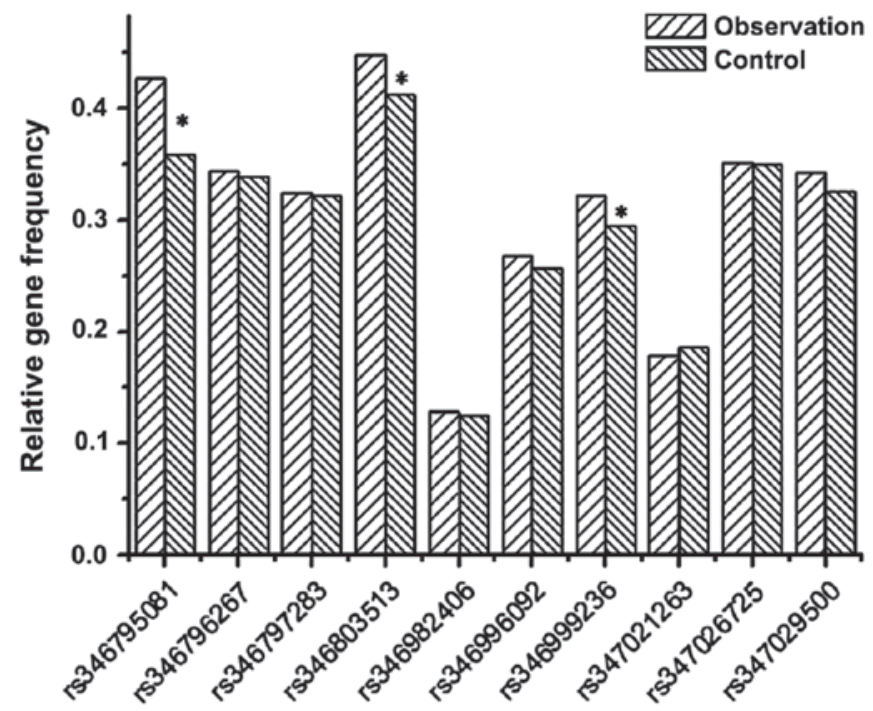

Figure 3. Difference in expression level averages at 10 SNP sites between the patient and control groups. *Significant difference between trait and control groups. SNPs, single nucleotide polymorphisms.

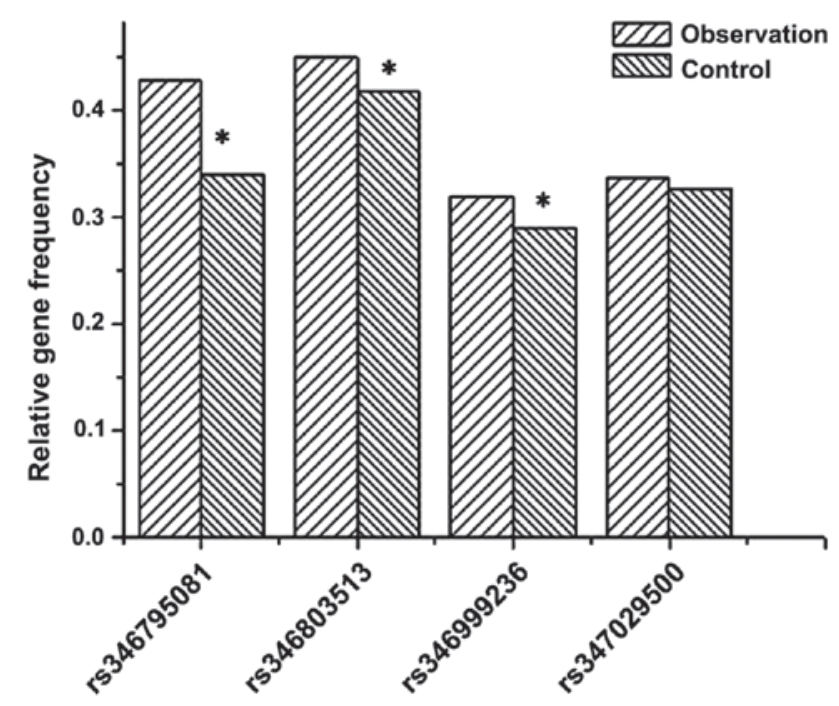

Figure 4. Allele frequency of four SNP sites between the patient and the control groups. "Significant differences between groups. SNPs, single nucleotide polymorphisms.

The final part of the study was concerned with the specific data analysis. This part was divided into two stages. The 1st stage comprised literature review and comparison with NCBI data bank. A total of 10 SNP sites that were likely to be correlated with PCOS were selected in order to ensure the efficiency of the analysis, few first conducted comparisons and analyses of DNA sequencing results within a small group of patients to exclude some irrelevant SNPs and facilitate the analysis. According to the results at this 1st stage, we analyzed the genomes of 200 PCOS patients and the control group and found that, among 10 SNP sites, only the alleles at rs346795081, rs346803513, rs346999236 and rs347029500 were significantly different. Differences at other SNP sites lacked statistical significance and were not taken into account for the next stage of the study. 
After exclusion of six SNP sites during the 1st stage, the experimental sample size was increased to 1,200 cases to verify the correlation between four SNP sites and PCOS. The results of the second analysis revealed no significant differences at the SNP rs347029500 site. However, the other three SNP sites, rs346795081, rs346803513 and rs346999236, were closely correlated with the occurrence of PCOS.

To conclude, three SNP sites were found to correlate closely with the occurrence of PCOS. This information can provide reference for further analysis and future studies on how specific genes regulate the onset and progress of PCOS.

\section{Acknowledgements}

This study was supported by the Changzhou Municipal Science and Technology Bureau (no. CJ20140021).

\section{References}

1. Trikudanathan S: Polycystic ovarian syndrome. Med Clin North Am 99: 221-235, 2015.

2. Bostanc1 MS, Akdemir N, Cinemre B, Cevrioglu AS, Özden S and Ünal O: Serum irisin levels in patients with polycysticovary syndrome. Eur Rev Med Pharmacol Sci 19: 4462-4468, 2015.

3. Al-Zubeidi H and Klein KO: Randomized clinical trial evaluating metformin versus oral contraceptive pills in the treatment of adolescents with polycystic ovarian syndrome. J Pediatr Endocrinol Metab 28: 853-858, 2015.

4. Yakubov Y and Mandel L: Bilateral parotid swelling in polycystic ovarian syndrome. J Oral Maxillofac Surg 74: 991-994, 2016.

5. Yang B1, Sun ZJ, Chen B, Zhang J, Zhao H, Li CW, Cao YK and Cao F: Statin ameliorates endothelial dysfunction and insulin resistance in Tibet women with polycystic ovary syndrome. Eur Rev Med Pharmacol Sci 20: 1185-1191, 2016.

6. Benk Silfeler D, Keskin Kurt R, Kaya OA, Yengil E, Hamamci B, Okyay AG and Beyazit A: Demodex folliculorum in polycystic ovary syndrome patients. Eur Rev Med Pharmacol Sci 19: 1141-1145, 2015.

7. Ting W, Yanyan Q, Jian H, Keqin H and Duan M: The relationship between insulin resistance and $\mathrm{CpG}$ island methylation of LMNA gene in polycystic ovary syndrome. Cell Biochem Biophys 67: 1041-1047, 2013.

8. Gondro C: Genome wide association studies. In: Primer to Analysis of Genomic Data Using R. Springer International Publishing, Cham, Switzerland, pp73-103, 2015.

9. Zhou X and Stephens M: Efficient algorithms for multivariate linear mixed models in genome-wide association studies. Nat Methods 11: 407-409, 2014
10. Zhou PP, Li Y, Ma ZD, Li ZY, Chen FY and Jiang YX: Single nucleotide polymorphisms in the promoter region of mir-133a-1 and in pre-mir-152 rs1707 may contribute to the risk of asthma in a Chinese Han population. Eur Rev Med Pharmacol Sci 20: 2642-2649, 2016.

11. Tasan M, Musso G, Hao T, Vidal M, MacRae CA and Roth FP: Selecting causal genes from genome-wide association studies via functionally coherent subnetworks. Nat Methods 12: 154-159, 2015.

12. Lerchbaum E, Schwetz V, Giuliani A and Obermayer-Pietsch B Assessment of glucose metabolism in polycystic ovary syndrome: HbAlc or fasting glucose compared with the oral glucose tolerance test as a screening method. Hum Reprod 28: 2537-2544, 2013

13. Legro RS, Arslanian SA, Ehrmann DA, Hoeger KM, Murad MH, Pasquali R and Welt CK; Endocrine Society: Diagnosis and treatment of polycystic ovary syndrome: An Endocrine Society clinical practice guideline. J Clin Endocrinol Metab 98: 4565-4592, 2013

14. Belosi C, Selvaggi L, Apa R, Guido M, Romualdi D, Fulghesu AM and Lanzone A: Is the PCOS diagnosis solved by ESHRE/ASRM 2003 consensus or could it include ultrasound examination of the ovarian stroma? Hum Reprod 21: 3108-3115, 2006.

15. Ecklund LC and Usadi RS: Endocrine and reproductive effects of polycystic ovarian syndrome. Obstet Gynecol Clin North Am 42: $55-65,2015$.

16. Abbott DH, Nicol LE, Levine JE, Xu N, Goodarzi MO and Dumesic DA: Nonhuman primate models of polycystic ovary syndrome. Mol Cell Endocrinol 373: 21-28, 2013.

17. Li R, Zhang Q, Yang D, Li S, Lu S, Wu X, Wei Z, Song X, Wang X, Fu S, et al: Prevalence of polycystic ovary syndrome in women in China: A large community-based study. Hum Reprod 28: 2562-2569, 2013.

18. Bili E, Dampala K, Iakovou I, Tsolakidis D, Giannakou A and Tarlatzis BC: The combination of ovarian volume and outline has better diagnostic accuracy than prostate-specific antigen (PSA) concentrations in women with polycystic ovarian syndrome (PCOs). Eur J Obstet Gynecol Reprod Biol 179: 32-35, 2014.

19. Mutharasan P, Galdones E, Peñalver Bernabé B, Garcia OA, Jafari N, Shea LD, Woodruff TK, Legro RS, Dunaif A and Urbanek M: Evidence for chromosome 2p16.3 polycystic ovary syndrome susceptibility locus in affected women of European ancestry. J Clin Endocrinol Metab 98: E185-E190, 2013.

20. Glueck CJ, Woo JG, Khoury PR, Morrison JA, Daniels SR and Wang P: Adolescent oligomenorrhea (age 14-19) tracks into the third decade of life (age 20-28) and predicts increased cardiovascular risk factors and metabolic syndrome. Metabolism 64: 539-553, 2015.

21. Bassiouny YA, Rabie WA, Hassan AA and Darwish RK: Association of the luteinizing hormone/choriogonadotropin receptor gene polymorphism with polycystic ovary syndrome. Gynecol Endocrinol 30: 428-430, 2014. 